\section{PTH-038 A NOVEL IMAGE ANALYSIS APPROACH FOR SEMIAUTOMATED OUANTIFICATION OF CYTOKINE DISTRIBUTION IN HUMAN COLONIC BIOPSIES}

doi:10.1136/gut.2011.239301.439

C Loetsch, ${ }^{1}$ G K Pot, ${ }^{2}$ J Sington, ${ }^{3} \mathrm{~J}$ R Dainty, ${ }^{1}$ E K Lund 1, * 'Institute of Food Research, Norwich, UK; ${ }^{2}$ Wageningen University, Wageningen, The Netherlands; ${ }^{3}$ Norfolk and Norwich University Hospital, Norwich, UK

Introduction The authors have previously shown that both mRNA and protein profiles of IL-1 $\beta$ and IL- 8 in biopsies taken from macroscopically normal colon tissue during colonoscopy are significantly different to those taken during sigmoidoscopy. The former procedure involves emptying of the bowel $24 \mathrm{~h}$ prior to sample collection while in the latter case the distal bowel is voided immediately before sample collection. The authors hypothesise that these changes are a result of exposure to the resident bacteria. The aim of this study was to develop computer-assisted immunohistochemical methodologies to investigate whether differences in cytokine profiles, associated with the presence of gut microbiota, are related to changes in the crypt or lamina propria.

Methods Biopsies from apparently healthy tissue $(\mathrm{n}=30)$ were taken at $20-30 \mathrm{~cm}$ from the anal verge, from the same patient, after both colonoscopy and sigmoidoscopy. Serial sections of colonic mucosa from all specimens were immunohistochemically stained to compare changes in protein levels of IL-1 $\beta$ and IL-8 in both crypts and lamina propria. Cytokine expression levels were measured by a computer-assisted quantification method using ImagePro Plus 6.0 analysis software. Segmentational thresholds were set up to classify the immunopositive area into strong, moderate and weak staining. Lamina propria and crypt tissue were individually selected using a drawing tool. Internal control samples from the same specimen, as well as positive (appendix, tonsil) and negative controls (isotype control), were analysed in each run. Negative controls showed no detectable staining within the segments used for analysis. All slides were individually evaluated by a histopathologist to compare the automated with the manual scoring.

Results The authors have effectively set up an objective computer-assisted measurement technique to analyse staining patterns of soluble cytokines. Changes in strong and moderate staining levels in lamina propria were significantly different from those in the crypts for both IL-1 $1 \beta$ and IL-8. Staining classified as moderate or strong was connected with specific cells in either the crypt or lamina propria. In contrast to gene expression and total protein for these cytokines the authors found no significant difference in total staining of IL- $1 \beta$ and only marginal differences in IL-8 staining in biopsies taken following different bowel preparations. 
Conclusion This semiautomated image analysis technique is able to detect different staining patterns of soluble cytokines like IL-1 $\beta$ and IL- 8 in individual compartments of the colonic mucosa and is therefore a potentially suitable tool for identification of more specific patterns of expression within the mucosa.

Competing interests None.

Keywords cytokines, image analysis, immunohistochemistry, mucosa. 